\title{
Condições higiênico-sanitárias em uma escola pública do município de Januária-MG antes e após o treinamento dos manipuladores de alimentos
}

\author{
Joseane Moreira dos Santos ${ }^{1}$, Luiz Carlos Ferreira ${ }^{2 *}$
}

\begin{abstract}
Resumo
O treinamento dos manipuladores de alimentos constitui um aspecto importante na promoção da segurança alimentar em estabelecimentos de preparo e manipulação de alimentos, evitando riscos à saúde dos consumidores. Os manipuladores de alimentos constituem um elemento primordial nos serviços de alimentação, sendo responsáveis por casos de contaminação dos alimentos, por isso e necessário orientar e treinar esses manipuladores sobre os cuidados necessários para a oferta de um alimento seguro do ponto de vista higiênico-sanitário. O presente trabalho objetivou avaliar a eficácia do treinamento de manipuladores de alimentos de uma cantina de escola pública do município de Januária-MG, visando à redução dos riscos à saúde para os consumidores. Inicialmente, foi utilizada uma lista de verificação (checklist) para avaliar as atuais condições higienicossanitárias da cantina da escola. Posteriormente, foi promovido um treinamento em boas práticas de manipulação de alimentos para os manipuladores de alimentos da cantina da escola avaliada. Por fim, foram avaliadas novamente as condições higienicossanitárias da cantina da escola com o objetivo de verificar a eficiência do treinamento realizado com os manipuladores. A qualidade da água de abastecimento da escola avaliada neste estudo também foi determinada. Após a primeira aplicação da lista de verificação antes do treinamento dos manipuladores, o estabelecimento avaliado apresentou um percentual de atendimento às Boas Práticas de Fabricação (BPF) de 74,5\%, sendo classificado como "regular". Após o treinamento, o estabelecimento avaliado apresentou um percentual de atendimento às Boas Práticas de Fabricação (BPF) de 81,8\%, passando a ser classificado como "bom". O treinamento promovido no estabelecimento avaliado possibilitou uma melhoria das condições higiênico-sanitárias, uma vez que, a classificação quanto ao atendimento às boas práticas variou de "regular" para "bom". A água utilizada pelo estabelecimento pesquisado estava fora dos padrões de potabilidade determinados pela legislação brasileira.
\end{abstract}

Palavras-chave: Alimentação Escolar. Segurança Alimentar. Qualidade da Água.

\section{Hygienic-sanitary conditions in a public school in the municipality of Januária-MG before and after the training of food handlers}

\begin{abstract}
The training of food handlers is an important aspect in promoting food safety in food preparation and handling establishments, while avoiding health risks to consumers. The present study aimed to evaluate the efficacy of the training of food handlers of a public school canteen in the city of Januária-MG, aiming at reducing health risks for consumers. This study was conducted in a public school located in the rural area of the municipality of Januária-MG. Initially, a checklist was used to evaluate the current hygienic and sanitary conditions of the school canteen. Subsequently, training in Good Manufacturing Practices (GMP) was promoted for the food handlers of the evaluated school canteen. Finally, the hygienic and sanitary conditions of the school canteen were evaluated again in order to verify the efficiency of the training performed with the manipulators. The quality of the school supply water evaluated in this study was also determined. After the first application of the checklist before the training of the manipulators, the evaluated establishment presented a percentage of Good Manufacturing Practices of 74.5\%, being classified as
\end{abstract}

${ }^{1}$ Instituto Federal Norte de Minas Gerais http://orcid.org/0000-0001-5954-2590

${ }^{2}$ Instituto Federal Norte de Minas Gerais.

http://orcid.org/0000-0001-6873-3567

*Autor para correspondência: luizcarlos2169@gmail.com

Recebido para publicação em 26 de março de 2019. Aceito para publicação em 07 de agosto de 2019.

e-ISSN: 2447-6218 / ISSN: 2447-6218 / (C) 2009, Universidade Federal de Minas Gerais, Todos os direitos reservados. 
dos Santos, M. J.; Ferreira, C. L.

"regular". After the training, the evaluated establishment presented a percentage of Good Manufacturing Practices attendance of $81.8 \%$, being classified as "good". The training promoted in the evaluated establishment allowed an improvement of the hygienic-sanitary conditions, since, the classification regarding the attendance of the good practices ranged from "regular" to "good". The water used by the researched establishment was outside of the potability standards determined by Brazilian legislation.

Keywords: School Feeding. Food Safety. Water Quality.

\section{Introdução}

As refeições produzidas em Unidades de Alimentação e Nutrição (UAN) escolares devem atender às necessidades nutricionais dos alunos, oferecendo-lhes, além de alimentos adequados nos aspectos sensoriais e nutricionais, produtos seguros em relação às condições higiênico-sanitárias (Boaventura et al., 2017). No ambiente escolar, é de suma importância à oferta de alimentos saudáveis, pois uma criança bem alimentada apresenta maior aproveitamento escolar e melhores condições para um crescimento e desenvolvimento saudáveis. O consumo de alimentos em condições inapropriadas pode influenciar negativamente a saúde dos alunos e desencadear o surgimento de Doenças Transmitidas por Alimentos (DTAs), levando a resultados negativos na aprendizagem (Porto et al., 2015).

A preparação de grande quantidade de alimentos, como ocorre em instituições de ensino na preparação da merenda escolar, implica em riscos para os consumidores, sendo de grande importância à utilização de medidas preventivas para a diminuição deste problema, por meio dos aspectos higiênico-sanitários no preparo do alimento e a informação sobre educação sanitária (Luz; Fortuna, 2015). Os manipuladores de alimentos constituem um elemento primordial nos serviços de alimentação, sendo responsáveis por casos de contaminação de alimentos, tanto por hábitos inadequados de higiene pessoal como lavagem das mãos, ou ainda, por serem portadores de microrganismos patogênicos. A inadequada manipulação é uma das principais fontes de contaminação, estando relacionada com a higienização incorreta para o exercício da função (Shinohara et al., 2016).

A contaminação dos alimentos durante a manipulação ocorre quando medidas higiênico-sanitárias não são adotadas e as condições ambientais são insatisfatórias para sua manipulação. A partir disso, é preciso orientar e treinar os manipuladores sobre os cuidados na aquisição, acondicionamento, manipulação, conservação e exposição ao consumo dos alimentos, bem como a estrutura física do local de manipulação (Boaventura et al., 2017).

A instrução quanto aos hábitos dos manipuladores é de fundamental importância para o controle dos riscos de contaminação dos alimentos, e a consequente prevenção de doenças transmitidas por alimentos (Shinohara et al., 2016). Os hábitos pessoais dos manipuladores são fator de grande importância com relação às condições higiênico-sanitárias (Paiva, 2015), sendo que a ausência de profissionais capacitados compromete a elaboração da documentação necessária, treinamento e supervisão dos manipuladores, assim como a implementação das boas práticas de produção (Santos et al., 2018). Deve-se ressaltar que o treinamento deve ser um processo contínuo e planejado, pois não é possível realizar mudanças estruturais sem que haja uma conscientização constante por parte dos manipuladores (Shinohara et al., 2016).

Levando em consideração a importância dos manipuladores de alimentos na transmissão de DTAs e a relevância da aplicação das boas práticas de manipulação na garantia de segurança dos alimentos, esse trabalho buscou avaliar a eficácia do treinamento de manipuladores de alimentos de uma cantina de escola pública do município de Januária-MG, visando à redução dos riscos à saúde para os consumidores.

\section{Material e métodos}

Inicialmente, foi utilizada uma lista de verificação (checklist) para avaliar as atuais condições higienicossanitárias da cantina da escola. A lista de verificação utilizada foi adaptada da proposta de lista de verificação constante da RDC no 275 de 21 de outubro de 2002 da Agência Nacional de Vigilância Sanitária (ANVISA, 2002). A lista de verificação foi composta por 55 itens divididos em 11 grupos: Higiene, Higienização das mãos, Higiene das mãos, Ambiente, Merendeira, Gêneros Alimentícios, Estoque, Controle de Pragas, Abastecimento de Água, Lixo e Instalações Sanitárias. O número de itens de cada grupo está descrito na Tabela 1. No grupo Higienização das mãos foram avaliados os itens: presença de lixeira acionada por pedal, papel toalha, lavatórios exclusivos para higienização das mãos, sabão líquido e água corrente. No grupo Higiene das mãos foram avaliados os itens: lavagem das mãos após troca de função, após contato com o lixo, após limpar, utilizar panos e material de limpeza, após tocar em alimentos crus ou não higienizados, entre manipulação de alimentos crus e cozidos e após entrar em contato com o uniforme, bancada etc.

A lista de verificação foi preenchida no próprio estabelecimento pelo pesquisador e cada item que apresentou conformidades, foi computado como SIM, os itens não conforme foram computados como NÃO e aqueles 
Condições higiênico-sanitárias em uma escola pública do município de Januária - MG antes e após o treinamento dos manipuladores de alimentos

não pertinentes à avaliação do estabelecimento foram computados como não aplicável (NA).

Tabela 1 - Número de itens que constitui os grupos da lista de verificação (Adaptado da resolução RDC no 275 da ANVISA de 2002)

\begin{tabular}{lc}
\hline \multicolumn{1}{c}{ Grupos } & $\begin{array}{c}\text { Número de itens } \\
\text { avaliados }\end{array}$ \\
\hline Higiene & 10 \\
Higienização das mãos & 05 \\
Higiene das mãos & 06 \\
Ambiente & 03 \\
Merendeira & 08 \\
Gêneros Alimentícios & 07 \\
Estoque & 06 \\
Controle de Pragas & 02 \\
Abastecimento de Água & 02 \\
Lixo & 02 \\
Instalações Sanitárias & 04 \\
\hline Total & $\mathbf{5 5}$ \\
\hline
\end{tabular}

Na contagem dos pontos foi atribuído o valor de 1 ponto para cada resposta SIM, sendo que, as respostas NÃO receberam nota zero. Para a avaliação global do estabelecimento as respostas NA foram diminuídas do total de itens, não sendo, portanto, computadas na soma final. Para classificar o estabelecimento quanto ao atendimento das Boas Práticas de Fabricação (BPF), consideramos a soma total dos pontos, referentes às respostas SIM, utilizando a seguinte equação (Eq. 1):

$$
\text { Atendimento }(\%)=\frac{\text { Total de SIM }}{\text { Total de itens }- \text { Itens } N A} \times 100
$$

Segundo a RDC no 275 de 21 de outubro de 2002 da ANVISA, os estabelecimentos podem ser divididos em três grupos, de acordo com os pontos obtidos na lista de verificação. A classificação dos respectivos grupos pode ser visualizada na Tabela 2 .

Tabela 2 - Classificação dos estabelecimentos de acordo com o número de itens atendidos na lista de verificação de Boas Práticas de Fabricação segundo a RDC no 275 de 21 de outubro de 2002 da ANVISA (ANVISA, 2002)

\begin{tabular}{lc}
\hline \multicolumn{1}{c}{ Classificação } & Pontuação (\%) \\
\hline Grupo 1 (bom) & De 76 a 100 \\
Grupo 2 (regular) & De 51 a 75 \\
Grupo 3 (ruim) & $\leq 50$ \\
\hline
\end{tabular}

Posteriormente, foi promovido um treinamento em boas práticas de manipulação de alimentos para os manipuladores de alimentos da cantina da escola avaliada. No treinamento foram realizados minicursos e oficinas sobre boas práticas de manipulação de alimentos. Por fim, foram avaliadas novamente as condições higienicossanitárias da cantina da escola com o objetivo de verificar a eficiência do treinamento realizado com os manipuladores.

A qualidade da água de abastecimento da escola avaliada neste estudo também foi determinada. O parâmetro microbiológico analisado para verificar a qualidade microbiológica da água foi a contagem de bactérias coliformes totais e a detecção da bactéria Escherichia coli. Para quantificação de bactérias coliformes totais e E. coli foi utilizado o método Colilert IDEXX Quanti-Tray ${ }^{\circledR} / 2000$. O Colilert, sistema patenteado por IDEXX Laboratories, que utiliza nutrientes (açúcares ligados a radicais orgânicos cromogênicos) que fazem com que os microrganismos de interesse presentes na amostra produzam uma mudança de cor (ou fluorescência) no sistema inoculado. O método Colilert contém os substratos cromogênico orto-nitrofenil- $\beta$-D-galactopiranosídeo (ONPG) e o fluorogênico 4-metilumbeliferil- $\beta$-D-glucoronídeo (MUG), que detectam simultaneamente as bactérias do grupo coliforme total e E. coli em amostras de água (Manafi, 2000).

\section{Resultados e discussão}

Após a primeira aplicação da lista de verificação antes do treinamento dos manipuladores, os percentuais de adequação em todos os itens analisados por grupo variaram entre 33,3 e 100\% (Tabela 3). Neste momento, o estabelecimento avaliado apresentou um percentual de atendimento às Boas Práticas de Fabricação (BPF) de $74,5 \%$, sendo classificado como "regular" (grupo 2) de acordo com a RDC 275 da Agência Nacional de Vigilância Sanitária. O percentual de atendimento para cada item avaliado está demonstrado na Tabela 3.

Os quesitos Lixo, Estoque e Gêneros Alimentícios foram os apresentaram maior índice de adequação (100\%), sendo classificados no grupo 1 (bom). O estabelecimento apresentava lixeiras tampadas e equipadas com pedal, além de serem fabricadas de material de fácil limpeza. Observou-se também que o lixo era separado em orgânicos e não orgânicos, estando livres de roedores e insetos.

O estoque estava organizado, com os produtos alimentícios acondicionados em prateleiras, espaçamentos necessários para ventilação e em local limpo. Os produtos estavam armazenados de acordo com cada tipo, identificados adequadamente e estavam dentro do prazo de validade. É necessário que as matérias-primas sejam armazenadas em local limpo e organizado, de forma a garantir proteção contra qualquer tipo de contaminantes e devem estar adequadamente acondicionados e identi- 
dos Santos, M. J.; Ferreira, C. L.

ficados, sendo que sua utilização deve respeitar o prazo

de validade (ANVISA, 2004).

Tabela 3 - Porcentagem de atendimento dos itens avaliados na lista de verificação (checklist)

\begin{tabular}{|c|c|c|c|c|}
\hline Itens & $\%$ de atendimento & $\begin{array}{c}\text { Bom } \\
76-100\end{array}$ & $\begin{array}{c}\text { Regular } \\
51-75\end{array}$ & $\begin{array}{l}\text { Ruim } \\
\leq 50\end{array}$ \\
\hline Higiene & 80 & $\mathrm{X}$ & & \\
\hline Higienização das mãos & 40 & & & $\mathrm{X}$ \\
\hline Higiene das mãos & 33,3 & & & $\mathrm{X}$ \\
\hline Ambiente & 66,6 & & $\mathrm{X}$ & \\
\hline Merendeira & 87,5 & $\mathrm{X}$ & & \\
\hline Gêneros alimentícios & 100 & $\mathrm{X}$ & & \\
\hline Estoque & 100 & $\mathrm{X}$ & & \\
\hline Controle de pragas & 50 & & & $\mathrm{X}$ \\
\hline Abastecimento de água & 50 & & & $\mathrm{X}$ \\
\hline Lixo & 100 & $\mathrm{X}$ & & \\
\hline Instalações sanitárias & 75 & $\mathrm{X}$ & & \\
\hline
\end{tabular}

No quesito Merendeira, foi verificado $87,5 \%$ de conformidade, se enquadrando no grupo 1 (bom). Foi observado o uso de adornos durante a manipulação, podendo comprometer a segurança dos alimentos. Magalhães et al. (2016) afirmam que o uso de adornos é considerado grande veículo de contaminação alimentar. Para Moura e Araújo (2012), o uso de adornos são práticas errôneas que podem carrear patógenos para os alimentos durante a manipulação. Paiva (2015) chama atenção para a necessidade do asseio para o trabalho com alimentos, ter unhas e cabelos cortados, não utilizar brincos, colares, anéis e maquiagem durante todo o tempo em que estiver trabalhando. $\mathrm{O}$ uso de anéis, relógios, brincos entre outros adornos e produtos cosméticos, não é permitido nos ambientes de produção de alimentos, pois se relacionam aos perigos físicos, químicos e biológicos (Aragão, 2016).

Os manipuladores usavam uniformes, toucas e calçados fechados. Não foi avaliada na cheklist a periodicidade na troca de uniformes dos manipuladores. De acordo com Garcia e Bassinello (2007), é necessário asseio pessoal, onde os uniformes devem ser trocados, no mínimo, diariamente e usados exclusivamente nas dependências internas do estabelecimento. Moura e Araújo (2012) alertam que os cabelos devem manter-se limpos e totalmente protegidos com rede ou touca ou outro acessório apropriado para este fim. Muller (2011) salienta que o manipulador de alimentos, quando executa sua higiene pessoal erroneamente e quando não se conduz por boas práticas de fabricação, é um fator de contaminação dos alimentos. Em linhas gerais, um ser humano sadio carrega consigo milhões de microrganismos por centímetro cúbico. Os hábitos pessoais dos manipuladores são fator de grande importância com relação às condições higiênico-sanitárias (Paiva, 2015).

Oliveira et al. (2008) afirmam que a adoção de técnicas corretas de manipulação dos alimentos e a conscientização dos profissionais envolvidos são fundamentais como medida de controle das infecções de origem alimentar, principalmente nos estabelecimentos que fornecem refeições coletivas. Uma manipulação incorreta e o descuido em relação às normas higiênicas favorecem a contaminação por microrganismos patogênicos (Mello et al., 2010).

No quesito Higiene, foi avaliado se a estrutura física da instituição estava em conformidade com a legislação vigente, sendo avaliados piso, parede, teto, portas e janelas, frequência de higienização de equipamentos e utensílios etc. O quesito apresentou $80 \%$ de conformidade nos itens avaliados, pois a cantina do estabelecimento não possuía telas de proteção nas janelas e nas portas. De acordo com a ANVISA (2004), as aberturas das áreas de armazenamento e preparação de alimentos devem conter telas de proteção para impedir o acesso de vetores e pragas, sendo que as mesmas precisam ser removíveis, de modo a facilitar a limpeza. As telas devem ter malha de $2 \mathrm{~mm}$, ser de fácil limpeza e em bom estado de conservação (ANVISA, 2004).

As Instalações Sanitárias apresentaram 75\% de conformidade, foi analisado se essas instalações eram separadas por sexo, se tinha serviço de água corrente e se as estruturas dessas instalações estavam em bom estado. Mesmo sendo classificado no grupo 1 (bom), alguns itens não estavam em conformidade. As portas e torneiras não possuíam fechamento automático, sen- 
Condições higiênico-sanitárias em uma escola pública do município de Januária - MG antes e após o treinamento dos manipuladores de alimentos

do inevitável o contato com os mesmos. As instalações sanitárias destinadas aos manipuladores da instituição pesquisada não eram separadas por sexo.

No quesito Ambiente foram analisados ventilação e circulação de ar, iluminação e instalação elétrica. O quesito apresentou $66,6 \%$ de conformidade nos itens avaliados. As instalações elétricas estavam em bom estado de conservação, as janelas da área de manipulação eram amplas, com isso se aproveitava ao máximo da iluminação natural para o desenvolvimento das atividades. Entretanto, a ventilação e circulação de ar não eram capazes de garantir o conforto térmico, como os raios solares incidiam diretamente na área de manipulação, os manipuladores afirmaram que o ambiente era muito quente.

O quesito Controle de Pragas apresentou 50\% de conformidade. A instituição pesquisada não apresenta janelas e portas com telas de proteção para o controle dessas pragas. Beux et al. (2013) afirmam que, no que tange ao controle de pragas, esse deve ser feita por uma empresa terceirizada contratada pelo estado e estar em conformidade com a legislação vigente. A RDC 216/2004 da Anvisa preconiza que devem ser implantados procedimentos de boas práticas de modo a prevenir ou minimizar a presença de insetos e roedores (ANVISA, 2004).

O Abastecimento de água apresentou 50\% de adequação. A instituição avaliada neste estudo não conta com abastecimento de água através de rede pública com tratamento prévio da água, sendo a água proveniente de poço artesiano, não existindo controle de potabilidade. A água utilizada para consumo direto ou no preparo dos alimentos deverá ser de qualidade, para isso deverá ser controlada periodicamente (Paiva, 2015). Scuracchio e Farache Filho (2011) destaca a importância de se tratar a água destinada ao consumo humano, devido a possibilidade de veicular grande quantidade de contaminantes físico-químicos e/ou biológicos.

No que ser refere ao reservatório de água, este estava revestido e livre de rachaduras, vazamentos e infiltrações, no entanto, o reservatório não é higienizado semestralmente. Segundo Rocha et al. (2011), instituições responsáveis por atender um grande público de pessoas como escolas utilizam água de seus reservatórios na produção de alimentos. Por isso, a higienização desses reservatórios deve ser realizada semestralmente ou sempre que necessário, por empresa especializada ou por funcionário da escola (SED, 2012), além de estar isentos de rachaduras e sempre tampados (Paiva, 2015).

A Higienização das Mãos e Higiene das Mãos foram os itens que apresentaram menor adequação com $40 \%$ e $33,3 \%$, respectivamente. No estabelecimento faltam toalhas de papel e sabonete líquido inodoro antisséptico para lavagem e secagem das mãos, e não existe informação do procedimento correto para a lavagem das mãos. Também não havia um lavatório na área de manipulação exclusivo para higienização das mãos, sendo o espaço utilizado para outras funções. Para Paiva (2015), higienizar as mãos é sem dúvida uma das melhores formas de prevenir a contaminação dos alimentos pelo manipulador. A higiene adequada das mãos pode diminuir o risco de contaminação dos alimentos por Staphylococcus aureus um dos principais patógenos envolvidos na intoxicação alimentar (Moreira et al., 2016).

Aragão et al. (2016) recomendam que devem existir lavatórios exclusivos para a higiene das mãos na área de manipulação, em posições estratégicas e número suficiente para a demanda, além disso, devem possuir sabonete líquido inodoro antisséptico ou sabonete líquido inodoro e um produto antisséptico, toalhas de papel não reciclado e coletor de papel sem contato manual. A lavagem das mãos é primordial para a manipulação dos alimentos, ela deve ser realizada constantemente e deve ser seguida da sanitização com solução de álcool $70 \%$ de modo a aumentar a eficácia do procedimento.

A presença de microrganismos, em elevada concentração, na superfície das mãos indica ineficiência nos procedimentos de antissepsia ou contaminação no contato com outras superfícies, o que acarretaria contaminação para o alimento em questão e consequente interferência na segurança do mesmo (Rodrigues et al., 2004). Os manipuladores não higienizavam as mãos com frequência ao entrar em contato com o uniforme, bancadas, entre manipulação de alimentos crus e cozidos e ao tocar em alimentos não higienizados. Moreira et al. (2012) afirmam que quando um alimento entra em contato com as mãos dos manipuladores, está sujeito à contaminação biológica, sendo necessário a frequente higienização das mãos.

Após a aplicação da lista de verificação pós-treinamento dos manipuladores, o item "higiene das mãos" sofreu alteração em relação a primeira lista de verificação, passando de $33,3 \%$ para $83,3 \%$ de atendimento, o que alterou a classificação deste item de "ruim" para "bom". Os manipuladores higienizavam as mãos com muito mais frequência ao entrar em contato com o uniforme, bancadas, e ao tocarem em alimentos não higienizados. Outro item que foi possível verificar alteração foi "merendeiras", variando de $87,5 \%$ para $100 \%$ de atendimento, não foi observado mais o uso de adornos por parte dos manipuladores, como verificado na primeira lista de verificação.

A melhoria nos dois itens citados demonstra a importância do treinamento para melhoria das condições higiênico-sanitárias do estabelecimento avaliado. Segundo a SED (2012), todos os funcionários de escola que entram em contato com alimentos devem ser qualificados tecnicamente nos requisitos mínimos de higiene pessoal, manipulação higiênica dos alimentos e doenças transmitidas por alimentos, através de cursos específicos e periódicos. Esses procedimentos devem ser observados nas instalações, equipamentos, móveis e utensílios; higienização das instalações; controle de pragas; abasteci- 
dos Santos, M. J.; Ferreira, C. L.

mento de água; manejo dos resíduos; cuidados a serem observados pelos manipuladores dos alimentos, como usar cabelos presos e protegidos por redes; cuidados com ingredientes e embalagens; preparo do alimento e armazenamento (Vasconcelos et al., 2007).

Após o treinamento, o estabelecimento avaliado apresentou um percentual de atendimento às Boas Práticas de Fabricação (BPF) de 81,8\%, passando a ser classificado como "bom" (grupo 1) de acordo com a RDC 275 da Agência Nacional de Vigilância Sanitária. Sendo assim, o treinamento promoveu uma melhoria nas condições higiênico-sanitárias do estabelecimento pesquisado. Os programas de treinamentos/capacitações de manipuladores enfatizam a importância da saúde individual e coletiva, incluem noções básicas de higiene pessoal e ambiental e destacam os danos que a ausência desses cuidados causa sobre a saúde do consumidor (Ribeiro et al., 2010).

Em relação a avaliação da qualidade microbiológica da água utilizada no estabelecimento pesquisado, a contagem de bactérias coliformes termotolerantes estavam acima do determinado na Portaria № 2914 de 12 de dezembro de 2011 do Ministério da Saúde, que dispõe sobre os procedimentos de controle e de vigilância da qualidade da água para consumo humano e seu padrão de potabilidade, definindo que, para uma amostra de $100 \mathrm{~mL}$, a água para consumo humano deve ser livre coliformes termotolerantes (MS, 2011). Não foi verificada a presença da bactéria E. coli nas amostras de água avaliadas. A qualidade da água é aspecto indispensável, quando se trata dos seus principais usos, em especial, para fins como o abastecimento humano (Souza et al., 2014).

A ingestão de alimentos contaminados com microrganismos, proveniente de água de má qualidade, pode tornar-se um problema gravíssimo para aqueles que fazem o consumo e, consequentemente, para os órgãos de saúde pública, uma vez que os gastos com o tratamento de doenças por ingestão de alimentos contaminados por bactérias do grupo coliforme são relativamente elevados (Rocha et al., 2011). Cardoso et al. (2007) salientam que quando utilizada na produção primária, processamento e preparo de alimentos, a água deve atender a exigências legais, de natureza físico-química e microbiológica, de modo a não expor a população a riscos.

\section{Conclusão}

O treinamento promovido no estabelecimento avaliado possibilitou uma melhoria das condições higiênico-sanitárias, uma vez que, a classificação quanto ao atendimento às boas práticas oscilou de "regular" (Grupo 2) para "bom" (Grupo 1) de acordo com RDC n 275/2002 da Agência Nacional de Vigilância Sanitária (ANVISA). A melhoria no atendimento aos itens do grupo Higienização das Mãos foi o fator responsável pela alteração da classificação do estabelecimento após o treinamento.

A água utilizada pelo estabelecimento pesquisado estava fora dos padrões de potabilidade determinados pela legislação brasileira, o que pode representar um risco para a saúde das pessoas atendidas pelo estabelecimento.

\section{Referências}

Agência Nacional de Vigilância Sanitária (ANVISA). 2002. Resolução RDC no 275. Disponível em: http://portal.anvisa.gov.br/ documents/10181/2718376/RDC_275_2002_COMP.pdf/fce9dac0ae57-4de2-8cf9-e286a383f254.

Agência Nacional de Vigilância Sanitária (ANVISA). 2004. Resolução RDC no 216. Disponível em: http://portal.anvisa.gov.br/ documents/33916/388704/RESOLU\%25C3\%2587\%25C3\%2583ORDC\%2BN\%2B216\%2BDE\%2B15\%2BDE\%2BSETEMBRO\%2BDE\% 2B2004.pdf/23701496-925d-4d4d-99aa-9d479b316c4b.

Aragão, T. F.; Celloni, I. S.; Matos, J. A. M. M.; Hespanhol, R. M. 2016. Avaliação do conhecimento dos profissionais envolvidos com a alimentação escolar do ensino publico sobre boas praticas de manipulação de alimentos. Revista Eletrônica de Educação da Faculdade Araguaia, 10: 121-124. Disponível em: http://www.faculdadearaguaia. edu.br/sipe/index.php/renefara/article/view/439/pdf_62.

Beux, J.; Primon, V.; Busato, M. A. 2013. Condições higiênicos sanitárias em local de produção e distribuição de alimentos em escolas públicas sob a ótica da produção mais limpa. Revista UNIFEBE, 1: 1-13. Diponível em: https://periodicos.unifebe.edu.br/index.php/ revistaeletronicadaunifebe/article/view/148.
Boaventura, L. T. A.; Frades, L. P.; Weber, M. L.; Pinto, B. O. S. 2017. Conhecimento de manipuladores de alimentos sobre higiene pessoal e boas práticas na produção de alimentos. Revista UNIVAP, 23: 53-62. Disponível em: https://revista.univap.br/index.php/revistaunivap/ article/view/1817.

Cardoso, C. V.; Almeida, R. C. C.; Guimarães, A. G.; Góes, J. A. W.; Silva, S. A.; Santana, A. A. C.; Huttner, L. B.; Vidal JR. P. O.; Figueiredo, K. V. N. A. 2007. Qualidade da água utilizada em escolas atendidas pelo Programa Nacional de Alimentação Escolar (PNAE), em Salvador-BA. Revista Instituto Adolfo Lutz, 66:287-291. Disponível em: https:// www.researchgate.net/publication/326621899_Qualidade_da agua_utilizada_em_escolas_atendidas_pelo_Programa_Nacional_de Alimentacao_Escolar_PNAE_em_Salvador-BA.

Garcia, D. M.; Bassinello, P. Z. 2007. Treinamento em boas práticas para manipuladores de alimentos. Embrapa Arroz e Feijão, Santo Antônio de Goiás, Goiás, Brasil. Disponível em: https://ainfo.cnptia.embrapa. br/digital/bitstream/CNPAF/26605/1/doc_202.pdf.

Luz, T. C. S.; Fortuna, J. L. 2015. Condições higiênico-sanitárias de manipuladores de merenda escolar de Instituições de ensino de Teixeira de Freitas-BA. Revista Eletrônica de Biologia, 8: 162-176. Disponível em: https://revistas.pucsp.br/index.php/reb/article/view/15463. 
Magalhães, J. A.; Carvalho, S. S.; Melquiades, R.; Rosa, G.; Merlini, L. S. 2016. Comércio ambulante de alimentos: condições higiênicosanitárias nos pontos de venda no município de Umuarama, Paraná, Brasil. Arquivos de Ciências Veterinárias e Zoologia da UNIPAR, 19: 147-152, 2016. Disponível em: http://revistas.unipar.br/index.php/ veterinaria/article/view/6087.

Manafi, M. 2000. Quantitative determinations of total coliforms and Escherichia coli in marine Waters with chromogenic and fluorogenic media. Journal of Applied Microbiology, 88: 280-285.

Mello, A. G.; Gama, M. P.; Marin, V. A.; Colares, L. G. T. 2010. Conhecimentos dos manipuladores de alimentos sobre boas praticas nos restaurantes públicos populares do estado do Rio de Janeiro. Brazilian Journal of Food Technology, 13: 60-68. Disponível em: http://bj.ital. sp.gov.br/artigos/html/busca/PDF/v13n1405a.pdf

Ministério da Saúde (MS). 2011. Portaria no 2914. Disponível em: http:// bvsms.saude.gov.br/bvs/saudelegis/gm/2011/prt2914_12_12_2011. html.

Moreira, A. M. F; Cruz, D. F; Abourihan, C. L. S. 2012. Atualização do manual de controle higiênico-sanitário, avaliação e capacitação dos manipuladores de alimentos em uma UAN de uma Instituição de longa permanência para idosos de Curitiba - PR. Cadernos da Escola de Saúde da UNIBRASIL, 6: 129-137. Disponível em: http://portaldeperiodicos. unibrasil.com.br/index.php/cadernossaude/article/view/2346.

Moreira B. G., PEDER, L. D., SILVA, C. M. 2016. Prevalência de Staphylococcus aureus nos vestíbulos nasais e mãos de manipuladores de alimentos em um hospital do oeste do Paraná, Brasil. Revista UNINGÁ Review, 28: 51-55. Disponível em: http://revista.uninga.br/index.php/ uningareviews/article/view/1846.

Moura, J. M. C., Araújo, K. C. P. 2012. Avaliação das boas práticas em UPRS de faculdades particulares da região metropolitana do Recife. Revista Conceito A, 3: 419-439. Disponível em: http://www. faculdadesaomiguel.com.br/pdf/revista-conceito/nutricao_avaliacaodas-boas-praticas.pdf.

Muller, I. M. Boas práticas de manipulação de alimentos com merendeiras. 2011. Disponível em: http://www.uniedu.sed.sc.gov.br/wp-content/ uploads/2013/10/Marcela-Ines-Muller.pdf.

Oliveira, M. N.; Brasil, A. L. D.; Taddei, J. A. A. C. 2008. Avaliação das condições higiênico sanitárias das cozinhas de creches públicas e filantrópicas. Ciência e Saúde Coletiva, 13: 1051-1060. Disponível em: http://www.scielo.br/scielo.php? script=sci arttext\&pid $=$ S1413-81232008000300028.

Paiva, E. P. 2015. Estudo da higiene e manipulação dos alimentos. 2015. Disponível em: https://sisacad.educacao. pe.gov.br/bibliotecavirtual/bibliotecavirtual/texto/ CadernodeRBHigieneeManipulaodosAlimentosRDDI.pdf.

Porto, E. B. S.; Schmitz, B. A. S.; Recine, E.; Carlos, M. D. L; Rodrigues, F. 2015. Condições higiênico-sanitárias das cantinas de escolas públicas e privadas do Distrito Federal - Brasil e seus fatores associados. Revista Visa em Debate, 3: 128-135. Disponível em:https://www.researchgate.net/ publication/286362253_Condicoes_higienico-sanitarias_das_cantinas de_escolas_publicas_e_privadas_do_Distrito_Federal_-_Brasil_e_seus_ fatores_associados.
Ribeiro, L. F., Argandon, E. J. S., Neto H. C. A., Macedo, P. P., Martis E. R. A importância da capacitação profissional dos manipuladores dos estabelecimentos alimentícios - Um estudo no município de Ivaiporã/ PR. 2010. Disponível em: http://www.abepro.org.br/biblioteca/ enegep2010_TN_STO_113_739_17380.pdf.

Rocha, E. S.; Rosico, F. S.; Silva, F. L.; Luz, T. C. S.; Fortuna, J. L. Análise microbiológica da água de cozinhas e/ou cantinas das instituições de ensino do município de Teixeira de Freitas (BA). 2011. Revista Baiana Saúde Pública, 34: 694-705. Disponível em: http://files.bvs. br/upload/S/0100-0233/2010/v34n3/a1871.pdf.

Rodrigues, M. M.; Bertin, B. M. A.; Assis, L.; Duarte, E. B.; Avelar, A. M. O.; Paixão, J. T. S.; Mattos, M. C; Souza, M. M. S. 2004. Indícios de Rotavirus na etiologia de um surto de infecção de origem alimentar. Revista Ciência e Tecnologia de Alimentos, 24: 88-93. Disponível em: http://www.scielo. $\mathrm{br} /$ scielo.php? script=sci_arttext\&pid=S0101-20612004000100017.

Santos, C. A. B., Santos, M. B., Dias, R. M. F. 2018. Avaliação do perfil higiênico-sanitário em unidades de alimentação e nutrição. Revista Ciência (In) Cena, 1: 101-113.

Scuracchio, P. A., Farache Filho, F. 2011. Qualidade da água utilizada para consumo em escolas e creches no município de São Carlos SP. Alimentação e Nutrição, 22: 641-647. Disponível em: https:// www2.fcfar.unesp.br/Home/Pos-graduacao/AlimentoseNutricao/ PaolaAndressaScuracchioME.pdf.

Secretaria de Estado de Educação (SED). Governo do Estado do Mato Grosso do Sul. Manual de Boas Práticas na Alimentação Escolar. 2012. Disponível em: http://www.sed.ms.gov.br/wp-content/ uploads/sites/67/2015/05/Manual-de-Boas-Pr\%C3\%A1ticas-naAlimenta\%C3\%A7\%C3\%A3o-Escolar.pdf.

Shinohara, N. K. S.; Almeida, A. A. M.; Nunes, C. G. P. S.; Lima, G. S.; Padilha, M. R. F. 2016. Boas práticas em serviços de alimentação: não conformidades. Revista Eletrônica Diálogos Acadêmicos, 10: 79-91. Disponível em: http://uniesp.edu.br/sites/_biblioteca/ revistas/20170627113500.pdf.

Souza, J. R.; Moraes, M. E. B.; Sonoda, S. L.; Santos, H. C. R. G. 2014. A Importância da qualidade da água e os seus múltiplos usos: Caso Rio Almada, Sul da Bahia, Brasil. Revista Eletrônica do Prodema, 8: 26-45. Disponível em: http://www.revistarede.ufc.br/rede/article/view/217.

Vasconcelos M. A. A., Castro A. M. V., Queiroz A. L. M., Araújo E. L. B., Nascimento G. S. M., Jesus I. A., Cabral T. M. A., Nascimento G. J. 2007. Qualidade higienico-sanitário de manipuladores de algumas indústrias de alimentos do Município de João Pessoa - PB. Disponível em: http://www.prac.ufpb.br/anais/IXEnex/iniciacao/documentos/ anais/7.TECNOLOGIA/7CTDTQAMT02.pdf. 
